DOI: $10.5277 /$ epe 160202

\author{
ANDRZEJ KRASIŃSKI ${ }^{1}$
}

\title{
SEPARATION OF OIL-IN-WATER EMULSIONS USING POLYMER COALESCENCE STRUCTURES
}

\begin{abstract}
Application of polymer media for coalescence of oil droplets and separation of O/W emulsion was studied. The research was focused on the structural design of a primary coalescence layer. High efficiencies of separation were obtained for structures made of finest fibres. The increase of the average fibre diameter led to a decrease of pressure drop and reduced efficiency of oil droplets removal. Thick fibres performed well as the drainage media and enabled detachment of large droplets. In addition the influence of the material wettability and resulting saturation of coalescence structures on their operation performance was studied.
\end{abstract}

\section{INTRODUCTION}

The separation of liquid-liquid dispersions is a very important process with numerous applications in various industries. Comparing to other separation methods, the coalescence filtration has many advantages such as high efficiency with regards to very fine droplets, reasonable energy requirements, no chemical additives to processed liquids, etc. Very often the presence of chemical compounds increases stability of the dispersion due to their surface activity. Surfactants adsorb on the liquid/liquid interface and contribute to additional repulsive interaction between droplets which significantly confines their approach. According to thermodynamic laws, a system tends to minimize its free energy, which means minimization of the interface area. Additional interactions resulting from presence of surfactants explain why no spontaneous coalescence and phase separation is observed in stable systems. In real processes, the surface active compounds are often present, either as additives or as typical compounds of processed liquids, and separation must be efficiently performed at such conditions (unless these conditions can be avoided, e.g. by means of adsorption to remove surfactants upstream the coalescer).

${ }^{1}$ Warsaw University of Technology, Faculty of Chemical and Process Engineering, ul. Waryńskiego 1, 00-645, Warsaw, Poland, e-mail: A.Krasinski@ichip.pw.edu.pl 
In the field of droplets coalescence in liquid-liquid systems, an extensive research has been done in last few decades [1-11]. A significant insight into process principles was provided in a review paper by Sherony et al. [2]. Many research studies were focused on identification of the effect of wettability on coalescence performance [1-4, $6-8,10,11]$. The conclusions were various, nevertheless in recent years predominates the opinion that good wettability by a dispersed phase liquid improves the process. Shin and Chase [8] published interesting results about improvement of the coalescence performance by adding polymer nanofibres to a fibre glass media. In fact, this concept was an incentive for presented research work. In most recent work in this field, Agarwal et al. [11] studied the effect of surface wettability on the coalescence performance by coating polyester (PET and PBT) and cellulose fibres with a nanoparticles. For effective separation of oil from water by so called surface filtration, characteristic for the membrane process, where oil droplets are retained on the influent side and do not pass through it, a super hydrophilic filter with size of pores smaller than the dispersed droplets is recommended. For effective separation of oil from water by means of the deep bed coalescence, a filter should be hydrophobic and oleophilic to provide high saturation of the porous structure. The use of relatively thick fibres is suggested in latter case to enhance the adhesion and droplets holdup within the filter.

Kundu and Mishra [12] studied the removal of emulsified oil from water using packed beds of resins. The commercial oleophilic resin Purolite OL 100 was applied as the coalescing medium tested against various operating conditions such as $\mathrm{pH}$, bed height, oil concentration and superficial velocity. Maiti et al. [13] investigated purification of oily wastewater by adsorption using a commercial resin. The efficiency lower than $90 \%$ of oil removal was achieved, however the process is limited with regards to inlet concentration and corresponding lifetime of adsorption bed.

At present, the highest efficiency of removal oil from water is achieved by membrane process. As presented in literature, modified membranes can efficiently separate submicron oil droplets, and are invulnerable to presence of surfactants. The major drawback is however fouling with solids as well as blocking pores with accumulated oil which adheres to the surface and reduces the flux. Zhu et al. [14] reviewed the progress and perspective of future developments of filtration membranes to overcome these limitations. Solomon et al. [15] demonstrated a novel design of hierarchical membranes with contrasting wetting properties with oil and water, which separated water-in-oil emulsions well below $1 \mu \mathrm{m}$ in drop size.

Patel and Chase [16] applied a superhydrophobic polypropylene fibrous membranes for separation of dispersed water from diesel. Their satisfactory results and conclusions are in-line with afore-mentioned findings that for high efficiency of surface filtration process, surface should not be wetted by dispersed phase liquid, i.e. water in this case.

This work is focused, however, not on the surface filtration which is typical of membrane separations but concerns deep bed coalescence process with drainage of enlarged droplets upstream the filter media. The novelty of the research includes fabrication of 
nonwovens made of one polymer material which includes only a fraction of nanofibres in the first layer to enhance the process efficiency, and simultaneously maintain the pressure drop on a reasonable level. Therefore, in this work, an effort is made to design reliable coalescers capable of handling such a "difficult" separation of stable O/W dispersions.

\section{MATERIALS AND METHODS}

Experimental setup. The main part of the experimental system consists of the stainless steel horizontal vessel, where coalescence structures were mounted in the baskettype holder. The photograph of the test rig is presented in Fig. 1.

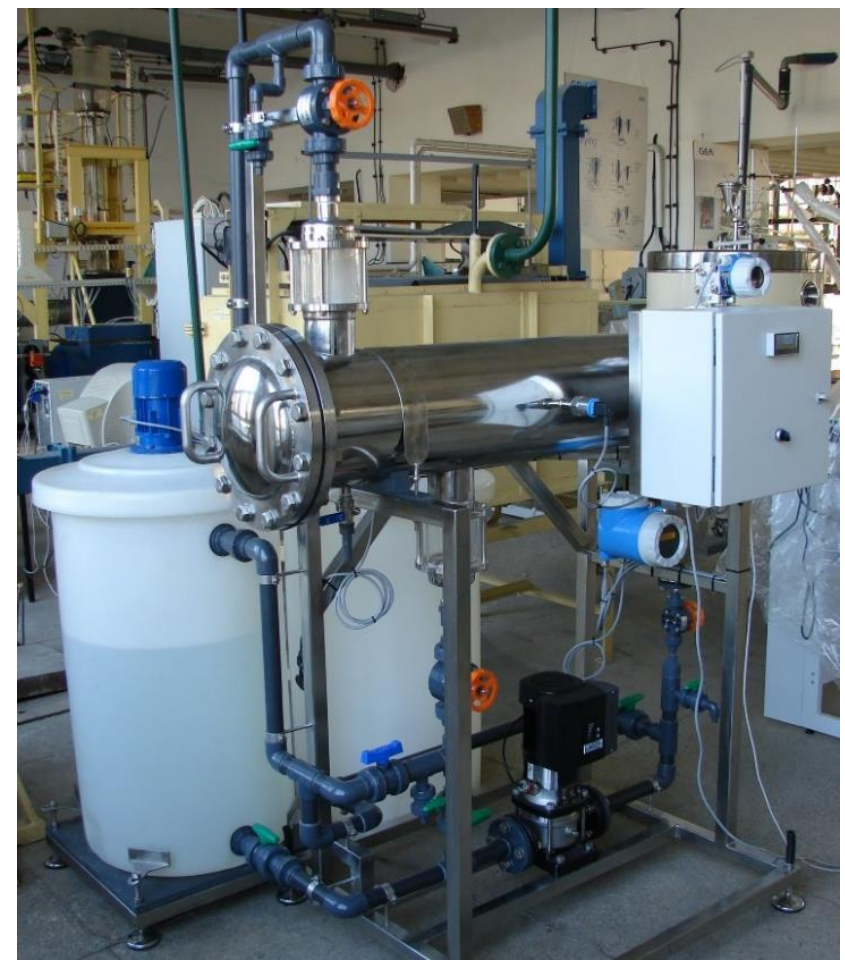

Fig. 1. The photograph of the test rig used in experiments

Test media in the form of flat panels with the diameter of $200 \mathrm{~mm}$ were used. The dispersion circulated in a closed loop from the tank, through the coalescence media mounted in the separator housing, and then back to the tank. The separated oil was collected in the upper glass vessel for interface level control. The diaphragm valves on the outlets enabled pressure control as well as keeping the interface level within the range 
of glass element. The emulsion was created in flow through the $0.37 \mathrm{~kW}$ multistage centrifugal pump (three impellers, speed $2870 \mathrm{rpm}$ ). Samples for analysis were collected after $60 \mathrm{~min}$ of operation in a steady state.

Test liquids. The dispersion of Shell V-Oil 1404 in RO water was used. The oil is a calibration fluid developed for testing diesel injectors. Therefore, its viscosity and density are well defined and correspond to specification for diesel fuel. However, it is highly additized with variety of compounds, which results in a low interfacial tension with water. The parameters such as density, surface and interfacial tensions for both liquids are listed Table 1.

Table 1

Properties of liquids used in experiments

\begin{tabular}{|c|c|c|c|}
\hline Liquid & Property ${ }^{\mathrm{a}}$ & Value & Instrument or data source \\
\hline \multirow{3}{*}{ RO water } & density, $\mathrm{kg} / \mathrm{m}^{3}$ & $\begin{array}{l}997 \\
997\end{array}$ & $\begin{array}{l}\text { Anton Paar DMA } 4500 \\
\text { Krüss EasyDyne K20 }\end{array}$ \\
\hline & surface tension, $\mathrm{N} / \mathrm{m}$ & $\begin{array}{l}7.03 \cdot 10^{-2} \\
6.72 \cdot 10^{-2} \\
6.81 \cdot 10^{-2} \\
\end{array}$ & $\begin{array}{l}\text { Sinterface Technologies DVA } 1 \\
\text { Krüss EasyDyne K20, plate method } \\
\text { Krüss EasyDyne K20, ring method }\end{array}$ \\
\hline & viscosity, $\mathrm{Pa} \cdot \mathrm{s}$ & $9.03 \cdot 10^{-4}$ & {$[17]$} \\
\hline \multirow{3}{*}{ Shell V-Oil 1404} & density, $\mathrm{kg} / \mathrm{m}^{3}$ & $\begin{array}{l}826 \\
817\end{array}$ & $\begin{array}{l}\text { Anton Paar DMA } 4500 \\
\text { Krüss EasyDyne K20 }\end{array}$ \\
\hline & surface tension, $\mathrm{N} / \mathrm{m}$ & $\begin{array}{l}2.75 \cdot 10^{-2} \\
2.74 \cdot 10^{-2} \\
2.67 \cdot 10^{-2} \\
\end{array}$ & $\begin{array}{l}\text { Sinterface Technologies DVA } 1 \\
\text { Krüss EasyDyne K20, plate method } \\
\text { Krüss EasyDyne K20, ring method }\end{array}$ \\
\hline & viscosity, $\mathrm{Pa} \cdot \mathrm{s}$ & $3.10 \cdot 10^{-3}$ & Product specification, mean value \\
\hline $\begin{array}{l}\text { RO water } \\
\text { /Shell V-Oil } 1404\end{array}$ & interfacial tension, $\mathrm{N} / \mathrm{m}$ & $\begin{array}{c}\begin{array}{c}4.48 \cdot 10^{-3} \\
6.30 \cdot 10^{-3} \\
\text { unsuccessful }\end{array}\end{array}$ & $\begin{array}{l}\text { Sinterface Technologies DVA } 1 \\
\text { Krüss EasyDyne K20, plate method } \\
\text { Krüss EasyDyne K20, ring method }\end{array}$ \\
\hline
\end{tabular}

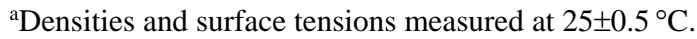

The measurements of surface and interfacial tensions using the drop volume method (refer to Sinterface Technologies DVA 1 in Table 1) give transient values of these properties. To recalculate them to stationary state an extrapolation method proposed by Göbel and Joppien [18] was applied. One can notice that the interfacial tension of the oil/water system is very low which is probably due to a high concentration of various additives in the calibration oil (e.g. corrosion inhibitors, lubricants etc.). Therefore, the water dispersion created by impeller of the centrifugal pump is characterised by very small oil droplets. The surface active compounds adsorb on the interface and are responsible for emulsion stabilization due to an additional interaction force between droplets as well as they increase elasticity of the interface. These two effects hinder the coalescence process and make separation very difficult. 
Measurements. The droplet size distribution of the dispersed oil were determined for inlet and outlet of the purified continuous phase using particle counters Spectrex PC2200, LiQuilaz SO2, and optical microscope Nikon E200 with phase contrast objectives. The total concentration of oil in water was determined using FTIR spectrometry (Nicolet iS 10) of the tetrachloroethylene extract [18]. Fractional efficiency was calculated from total oil concentrations and droplet size distributions on inlet and outlet of water.

Coalescence media. The separation of oil droplets from water using the coalescence media made of polymer fibres was studied. The design concept of the sandwiched multilayer coalescence media is schematically shown in Fig. 2. The research was focused mainly on the effect of primary coalescence structure design on the separation performance, i.e. efficiency and pressure drop. To capture all emulsion droplets this layer of porous media (I in Fig. 2) usually consists of fine, dense packed fibres, which should be wetted by oil.
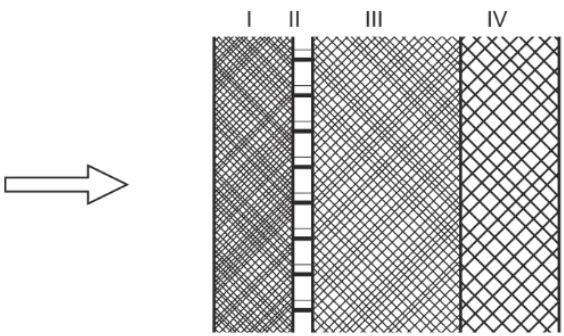

Fig. 2. Scheme of the multilayer coalescence structure:

I - primary coalescence layer, II - support plate, III - intermediate (secondary coalescence) layer, IV - drain layer

Downstream the inlet layer, a secondary coalescence structure is located (III in Fig. 2). Its role includes further growth of oil droplets, which disengage from upstream media. Due to high pressure drop and resulting forces, a perforated plate (II in Fig. 2) is mounted downstream the primary coalescence structure to support it and avoid compression of loose layers III and IV. The design of secondary coalescence layer (III) was the same in all experiments. The latter structure - drain layer (IV) - was mounted on the outlet. Its role includes the detachment of accumulated oil in form of large droplets of a uniform size.

Fibrous structures in the form of flat panels were manufactured using the technique called meltblow. During the process the polymer is molten, and then simultaneously mixed and extruded through a multichannel nozzle. The liquid jets formed at the nozzle outlet are elongated by the pressurized hot air, and subsequently solidify forming thin fibres. The fibres are collected on a receiver (e.g. rotating support core of a cartridge filter or a drum). Flat panels used in experiments were fabricated from a plain mat col- 
lected on a $300 \mathrm{~mm}$ diameter drum. The properties of fibrous structures depend on operating parameters such as the temperature of extruded polymer, its flow rate, temperature and local velocity of hot air, nozzle geometry and its location with regards to the receiver and the outlet of hot air duct. A combination of these parameters enables one to obtain the desired morphology of porous medium. The operating conditions can be indigenously programmed and automatically changed during the process to achieve gradients of porosities as well as different fibre diameters depending on the position in depth in a single manufacturing cycle.

In this work, fibrous mats made of three following polymers applied as a primary coalescence layer were experimentally studied: polypropylene, polyester (PBT, which stands for polybutylene terephthalate) and polyamide (Nylon 6,12). The effort was made to obtain similar structures made of different polymers but it was very difficult to achieve because the meltblow technology is sensitive to properties in molten state (e.g. MFI - melt flow index), which strongly affects the resulting size distribution of fibres in produced structures. In Table 2, the structural characteristics of fibrous mats have been presented.

Table 2

Specification of the materials used in experiments

\begin{tabular}{|c|c|c|c|c|c|c|}
\hline \multirow[b]{2}{*}{ Structure } & \multirow[b]{2}{*}{ Polymer } & \multirow[b]{2}{*}{$\begin{array}{c}\text { Commercial } \\
\text { name }\end{array}$} & \multicolumn{3}{|c|}{ Fibre diameter $[\mu \mathrm{m}]$} & \multirow{2}{*}{$\begin{array}{l}\text { Approximate } \\
\text { porosity } \\
\text { [\%] }\end{array}$} \\
\hline & & & Mean & $\begin{array}{l}\text { Standard } \\
\text { deviation }\end{array}$ & Size range & \\
\hline PP1 & \multirow{4}{*}{ polypropylene } & \multirow{4}{*}{$\begin{array}{l}\text { Borealis } \\
\text { Borflow } \\
\text { HL504FB }\end{array}$} & 1.37 & 1.02 & $0.37-7.15$ & $79.8 \pm 3.2$ \\
\hline PP2 & & & 11.6 & 6.25 & $4.11-26.5$ & $84.1 \pm 2.9$ \\
\hline PP3 & & & 16.7 & 6.25 & $7.83-36.5$ & $84.2 \pm 4.2$ \\
\hline PP4 & & & 59.5 & 14.2 & $31.4-87.0$ & $92.2 \pm 3.6$ \\
\hline PBT1 & \multirow{2}{*}{ polyester } & \multirow{2}{*}{$\begin{array}{l}\text { Celanex } \\
\text { PBT2008 }\end{array}$} & 1.64 & 1.03 & $0.85-10.5$ & $81.0 \pm 1.8$ \\
\hline PBT2 & & & 10.5 & 6.42 & $2.30-20.6$ & $85.3 \pm 3.2$ \\
\hline PA1 & \multirow{2}{*}{ polyamide } & \multirow{2}{*}{$\begin{array}{l}\text { Schulamid } \\
6 \mathrm{NV} 12\end{array}$} & 4.28 & 2.97 & $1.43-17.9$ & $81.4 \pm 2.6$ \\
\hline PA2 & & & 11.3 & 5.71 & $4.05-43.1$ & $84.2 \pm 3.5$ \\
\hline
\end{tabular}

The statistics of the fibre size distribution was obtained from digital analysis of SEM microphotographs (examples in Fig. 3). The porosity was estimated based on the bulk density (mass divided by approximate volume of the porous structure).

Capillary rise test. To characterize the wettability of selected polymers by process liquids the capillary rise tests with Shell V-Oil 1404 were carried out. Moreover, the afore mentioned results were correlated with literature data of surface energy of the media. Although criticism of the capillary rise test for significant simplifications, it is widely used to characterize wettability of porous media. Based on the modified LucasWashburn equation proposed by Siebold et al. [20]: 


$$
m^{2}=C \frac{\rho^{2} \sigma \cos \theta}{\mu} t, \quad C=\frac{\left(\pi R^{2} \varepsilon\right)^{2} r}{2 \xi^{2}}
$$

the slope of the linear dependence between square mass and time at the initial test interval was determined. In Equation (1), $m$ stands for mass in grams, $t$ time in seconds, $\rho$ liquid density in $\mathrm{kg} / \mathrm{m}^{3}, \sigma$ surface tension in $\mathrm{N} / \mathrm{m}$, and $\mu$ dynamic viscosity in Pa.s, respectively. The constant $C$ includes all considered geometrical parameters of the porous structure such as: $R$ test vessel radius, $\varepsilon$ porosity, $r$ equivalent pore radius, and $\xi$ tortuosity of pores.
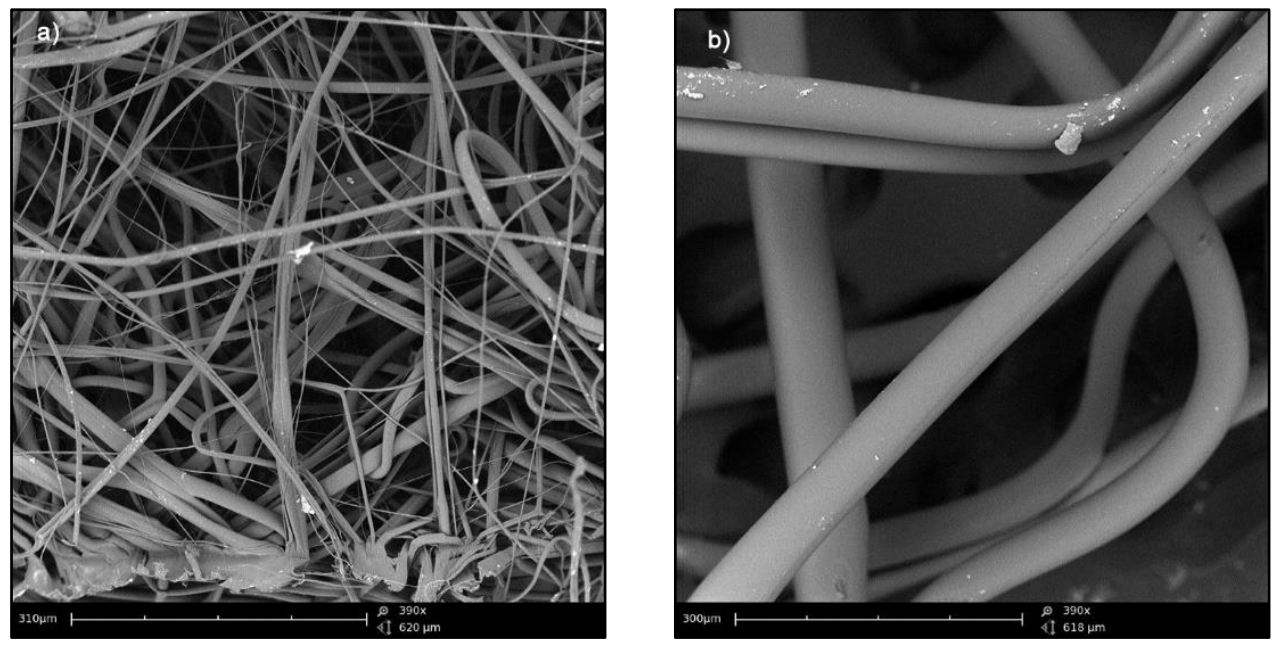

Fig. 3. Exemplary SEM microphotographs of fibrous media: a) PP2, b), PP4

The results show, however, that the rate of capillary transport during the test strongly depends on the media design, i.e. the fibre diameter and packing factor (or porosity), which affects the pore size. Hence, a representative comparison of the wettability by this method can be obtained for similar properties of the fibrous media, i.e. for similar values of proportionality constant $C$ in Eq. (1). To avoid this limitation, one can carry out analogical experiment for the same media with water as a test liquid and calculate so called "lipophilic-to-hydrophilic ratio" parameter (LHR) [21]:

$$
L H R=\frac{\cos \theta_{o}}{\cos \theta_{w}}=\frac{\left(\frac{m^{2}}{t}\right)_{o}}{\left(\frac{m^{2}}{t}\right)_{w}} \frac{\mu_{o}}{\mu_{w}} \frac{\rho_{w}^{2} \sigma_{w}}{\rho_{o}^{2} \sigma_{o}}
$$


where subscripts $o$ and $w$ refer to oil and water, respectively. LHR reflects a relative wettability of media by oil and water, and is a very useful parameter for characterisation of liquid/liquid coalescence media. However, it was impossible to perform such test with water for polypropylene due to its hydrophobicity.

Separation experiments. Experimentally, the influence of the design of the primary coalescence layer on separation performance has been examined as well as the effect of the properties of the drain layer on the size of detached droplets.

The secondary coalescence layer was the same in all experiments and consisted of PP3 media with the grammage of $300 \mathrm{~g} / \mathrm{m}^{2}$. The drain layer was made of PP4 (the grammage of $400 \mathrm{~g} / \mathrm{m}^{2}$ ), except few experiments when significantly thinner polypropylene and polyamide fibres were used to confirm the effect of its design and surface properties on the size of detached droplets. The separation efficiency in the test rig is not affected by the size of coalesced droplets provided they are large enough to reach a minimum terminal floating velocity. The dimensions of the separator housing and operating conditions guarantee a large safety margin in terms of the residence time in the settling compartment. However, in the case of testing various configuration of the drain layer, the separation efficiency was not of the importance - the research was focused on the size of coalesced droplets. The performance of various designs of coalescence structures listed in Table 2 were tested in variety of configurations and operating conditions as follows:

- primary coalescence layer - polypropylene: PP1, PP2, thickness ranging approximately from 1 to $6 \mathrm{~mm}$, which corresponds to the grammage ranging from 200 to $1000 \mathrm{~g} / \mathrm{m}^{2}$,

- primary coalescence layer - polyester: PBT1, PBT2: 1-5 mm $\left(200-1000 \mathrm{~g} / \mathrm{m}^{2}\right)$,

- primary coalescence layer - polyamide: PA1, PA2, 1-7 mm (200-1000 g/m²).

- inlet oil concentration: $12 \mathrm{~g} / \mathrm{dm}^{3}$ (ca. 1.45 vol. \%) in all experiments,

- flow per area: $7.2-36 \mathrm{~m}^{3} /\left(\mathrm{m}^{2} \cdot \mathrm{h}\right)$, which corresponds to superficial velocity $2-10 \mathrm{~mm} / \mathrm{s}$,

- dispersed oil: Shell V-Oil 1404,

- continuous phase: RO water.

\section{RESULTS AND DISCUSSION}

\subsection{DETERMINATION OF THE SURFACE PROPERTIES OF FIBRES}

To characterise the fibrous media in terms of surface wettability the capillary rise tests were carried out. The results are presented in Table 3 . The results correspond to literature values of the surface free energy for tested polymers [22]: $\mathrm{PP}-30.2 \mathrm{~mJ} / \mathrm{m}^{2}$, $\mathrm{PBT}-46.3 \mathrm{~mJ} / \mathrm{m}^{2}$, Nylon $6.12-67.0 \mathrm{~mJ} / \mathrm{m}^{2}$. 
Table 3

Results of wetting kinetics in the capillary rise experiments

\begin{tabular}{|l|c|c|c|c|c|}
\hline Structure & $\begin{array}{c}\text { Geometric } \\
\text { mean diameter } \\
{[\mu \mathrm{m}]}\end{array}$ & $\begin{array}{c}\text { Approximate } \\
\text { porosity } \\
{[\%]}\end{array}$ & $\begin{array}{c}\left(\Delta m^{2} / t\right)_{o} \\
{\left[\mathrm{~g}^{2} / \mathrm{s}\right]}\end{array}$ & $\begin{array}{c}\left(\Delta m^{2} / t\right)_{w} \\
{\left[\mathrm{~g}^{2} / \mathrm{s}\right]}\end{array}$ & $L H R$ \\
\hline PP1 & $1.37 \pm 1.02$ & $79.8 \pm 3.2$ & $0.316 \pm 0.112$ & - & - \\
\hline PBT1 & $1.64 \pm 1.03$ & $81.0 \pm 1.8$ & $0.275 \pm 0.073$ & $0.0176 \pm 0.0067$ & 172.3 \\
\hline PP2 & $11.6 \pm 6.25$ & $84.1 \pm 2.9$ & $1.523 \pm 0.326$ & - & - \\
\hline PBT2 & $10.5 \pm 6.42$ & $85.3 \pm 3.2$ & $1.423 \pm 0.302$ & $0.0654 \pm 0.0082$ & 240.0 \\
\hline PA2 & $11.3 \pm 5.71$ & $84.2 \pm 3.5$ & $1.012 \pm 0.175$ & $0.117 \pm 0.052$ & 95.4 \\
\hline
\end{tabular}

\subsection{SEPARATION EXPERIMENTS}

The coalescence filters were tested in separation experiments with $\mathrm{O} / \mathrm{W}$ dispersion created in flow through the centrifugal pump at its maximum speed $2870 \mathrm{rpm}$. The average size of oil droplets was around $4 \mu \mathrm{m}$ (Fig. 4).

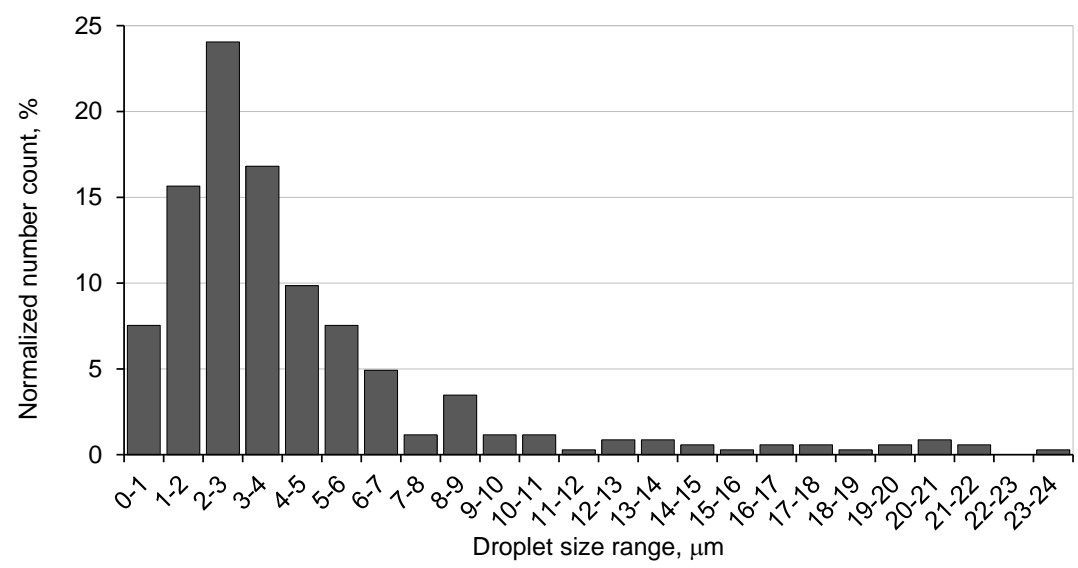

Fig. 4. Size distribution of oil droplets on the inlet

\subsubsection{EFFECT OF PRIMARY COALESCENCE LAYER DESIGN}

In Figures 5 and 6, the effects of the fibre size and bed depth for polypropylene media are presented. Even for the lowest grammage (or thickness) of PP1 equal to $200 \mathrm{~g} / \mathrm{m}^{2}$ the measured oil concentration on the outlet is clearly lower than for $1000 \mathrm{~g} / \mathrm{m}^{2}$ PP2 media. This can be explained by the larger total surface area which however causes a higher pressure drop. One can notice that there is almost no further increase of the separation efficiency above a certain thickness of primary coalescence structure. 

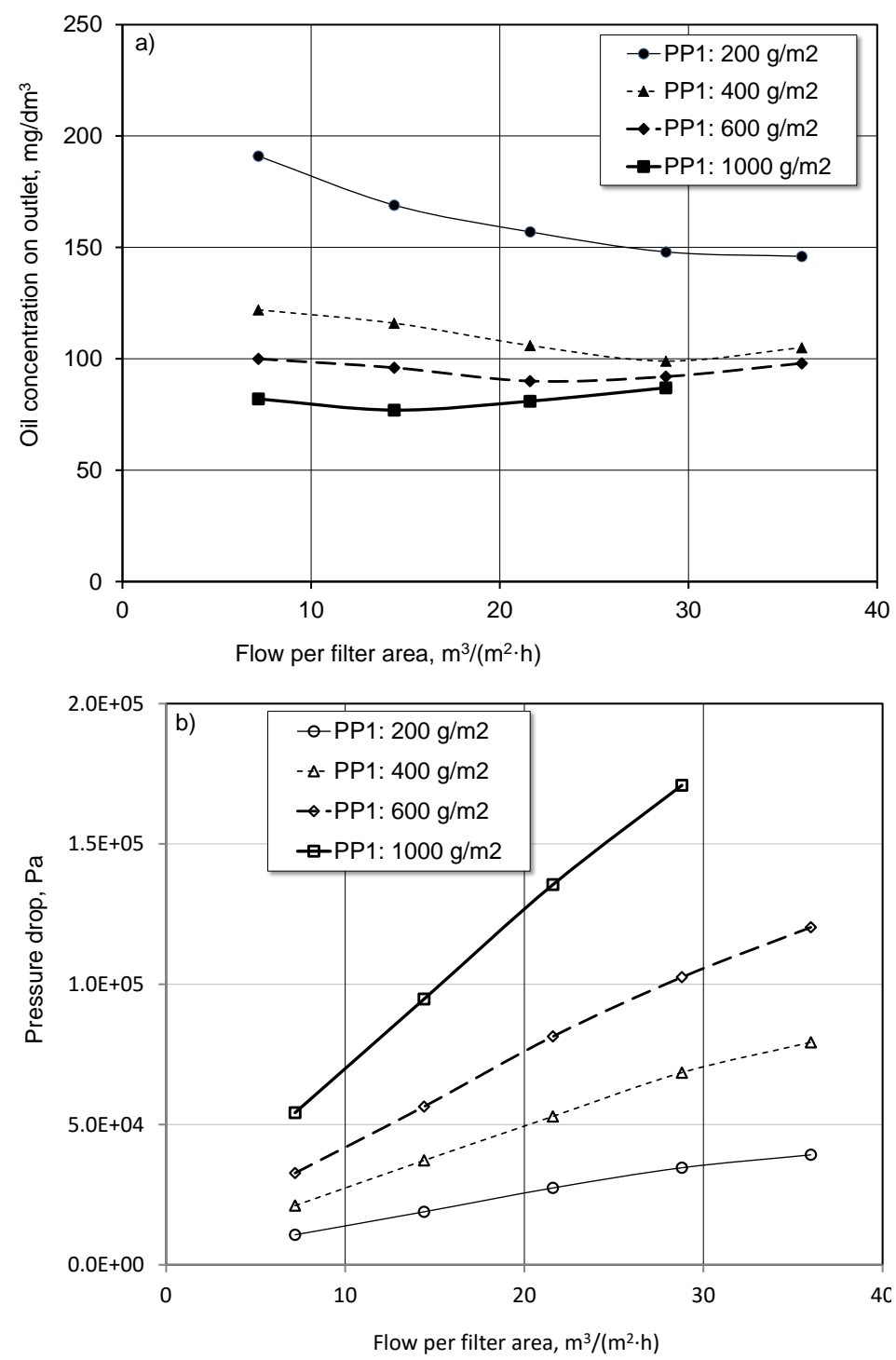

Fig. 5. Outlet oil concentration and pressure drop for the coalescence layer made of PP1

For thick fibre media, i.e. PP2, this "critical" bed depth is lower than in the case of PP1. Another difference between these structures is an opposite trend of changes in oil concentration depending on the flow rate. A slight increase of oil concentration is observed in the considered range of superficial velocities for PP2, which can potentially be explained by enhanced jetting and re-dispersion of accumulated oil. However, for PP1 the increase of flow rate brings about a decrease of outlet oil concentration for 200$400 \mathrm{~g} / \mathrm{m}^{2}$. 

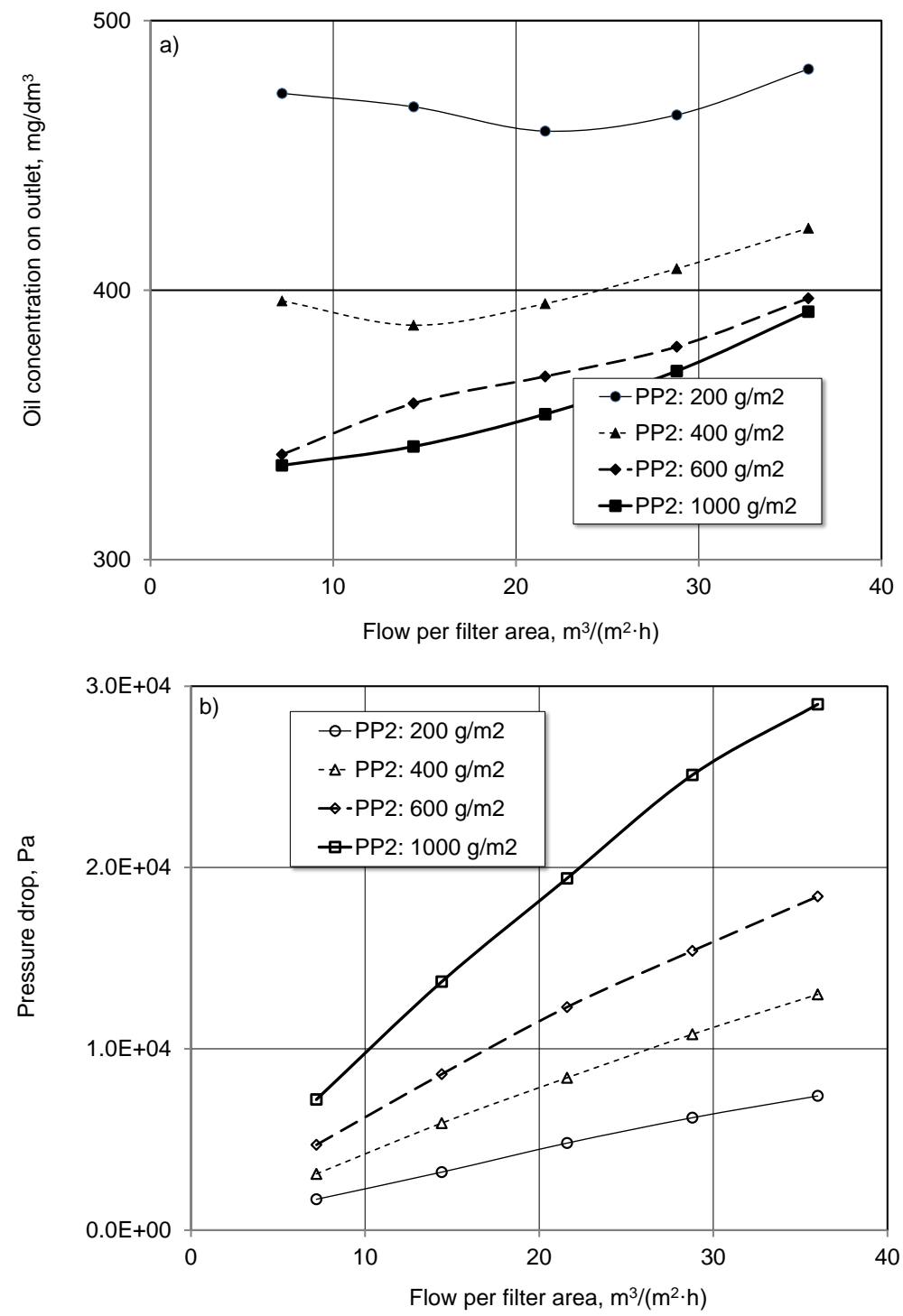

Fig. 6. Outlet oil concentration and pressure drop for coalescence layer made of PP2

This structure is relatively soft, hence a compression resulting from its weak dynamic stability can explain the observed trend. For the grammage of 600 and $1000 \mathrm{~g} / \mathrm{m}^{2}$ the $\Delta P$ is probably high enough to compress the fibrous media even at low flow rates. The lowest outlet oil concentration was obtained for $1000 \mathrm{~g} / \mathrm{m}^{2}$ PP1 structure (Fig. 5) - the cut-off diameter $d_{90}$ which represents the droplet size removed with efficiency 90\% was ca. $1.7 \mu \mathrm{m}$ (independently of flow rate). 

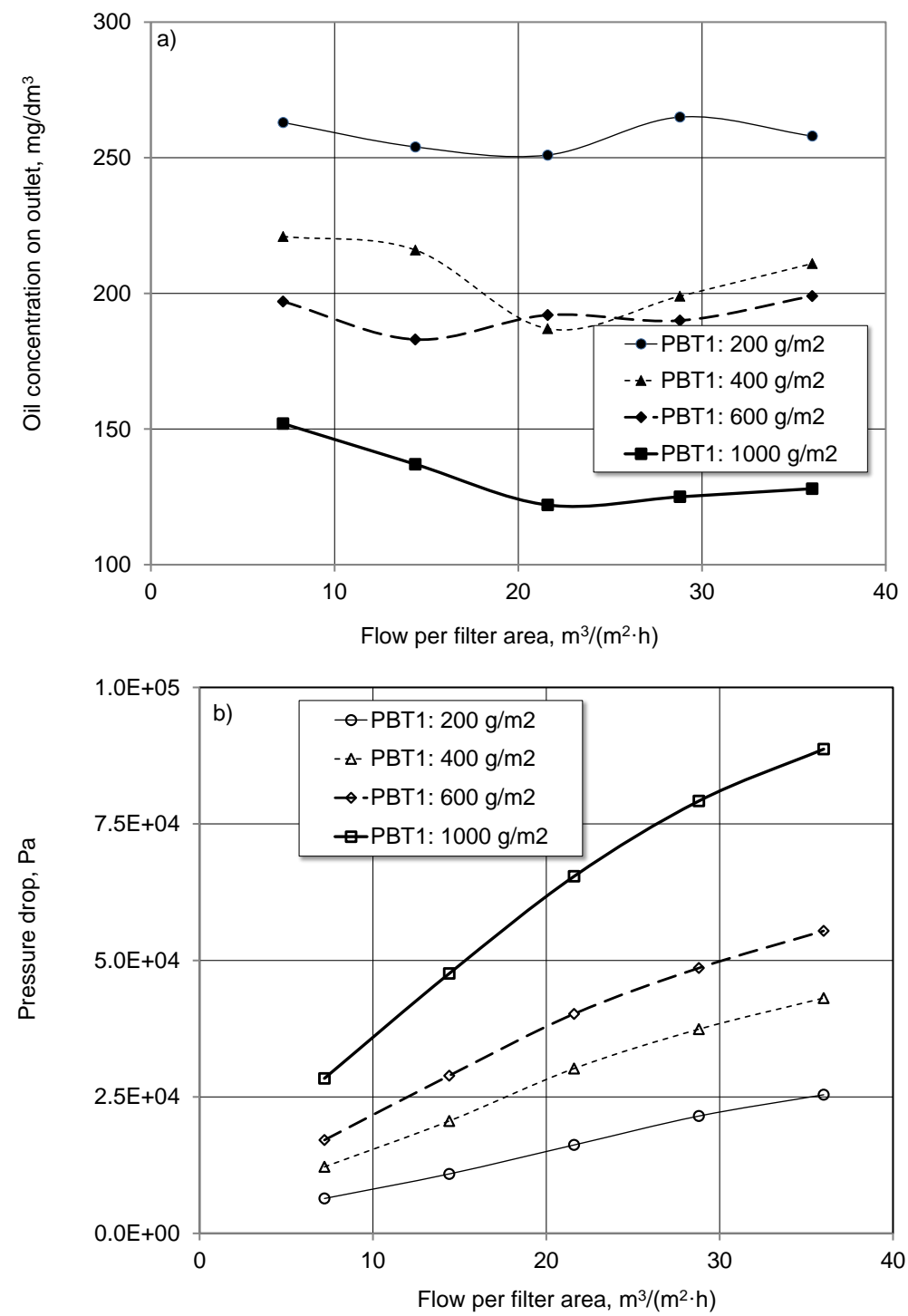

Fig. 7. Outlet oil concentration and pressure drop for coalescence layer made of PBT1

In Figures 7-10, the results of separation performance for coalescence media made of polyester and polyamide are presented. The structures with thinner fibres achieved a lower oil concentration in the effluent, however the pressure drop was significantly higher in these cases. The coalescence packing made of PBT1 characterised by a very similar morphology to PP1 performed significantly worse than polypropylene PP1 structure. However, the pressure drop for PBT1 was approximately a half of value obtained for the PP1 in all corresponding experiments (Figs. 5 and 7). 

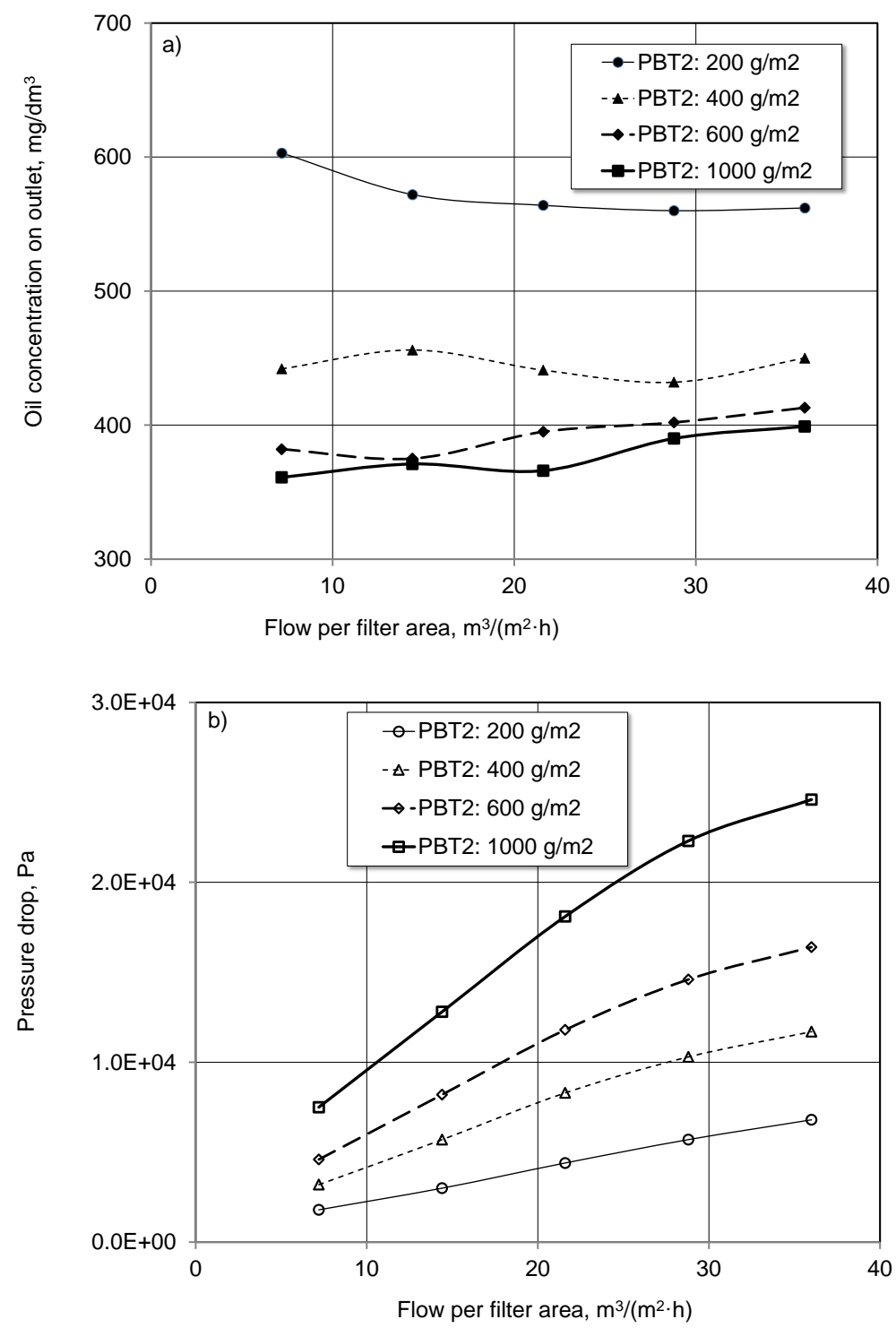

Fig. 8. Outlet oil concentration and pressure drop for coalescence layer made of PBT2

Observed effects can be explained by a good wettability of polypropylene with oil. In addition a high specific surface area and tight pores contribute to a very high filter saturation (or plugging), which on the other hand is beneficial for capturing small droplets. When comparing PBT2 to PP2 (Figs. 6 and 8) a higher separation efficiency was again obtained for polypropylene fibres, but in this case the difference in pressure drop was not very significant. 

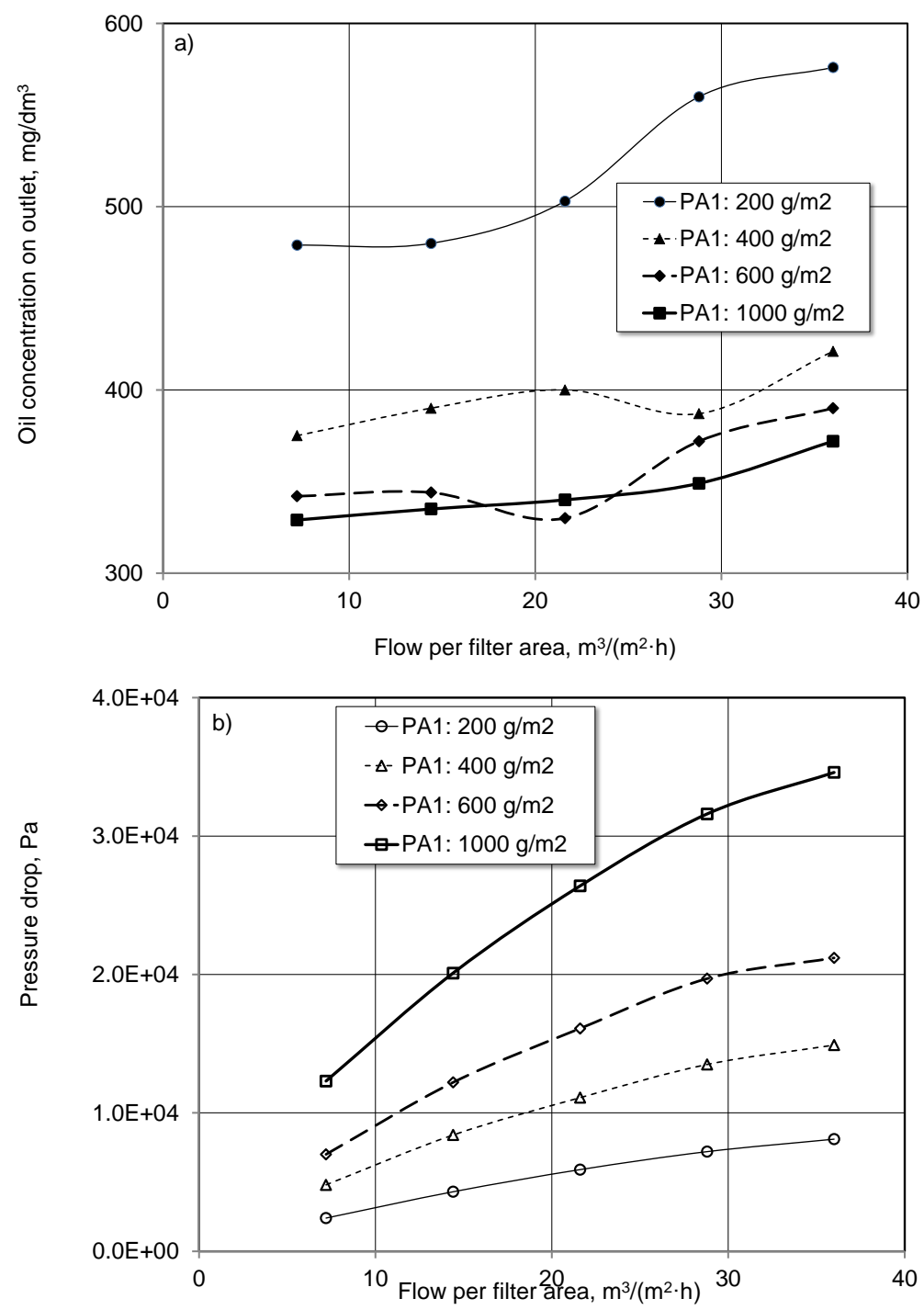

Fig. 9. Outlet oil concentration and pressure drop for coalescence layer made of PA1

In experiments with polyamide structures, the separation efficiency was relatively low comparing to media of similar morphology but made of different polymers (Figs. 6, 8 and 10). Due to different properties in melt, it was impossible to fabricate the polyamide structure similar to PP1 and PBT1 using the meltblow technique. This polymer is very tough in processing and requires rigorous pretreatment conditions but even then the morphology of PA1 did not approach fibre size obtained for PP1 and PBT1. 

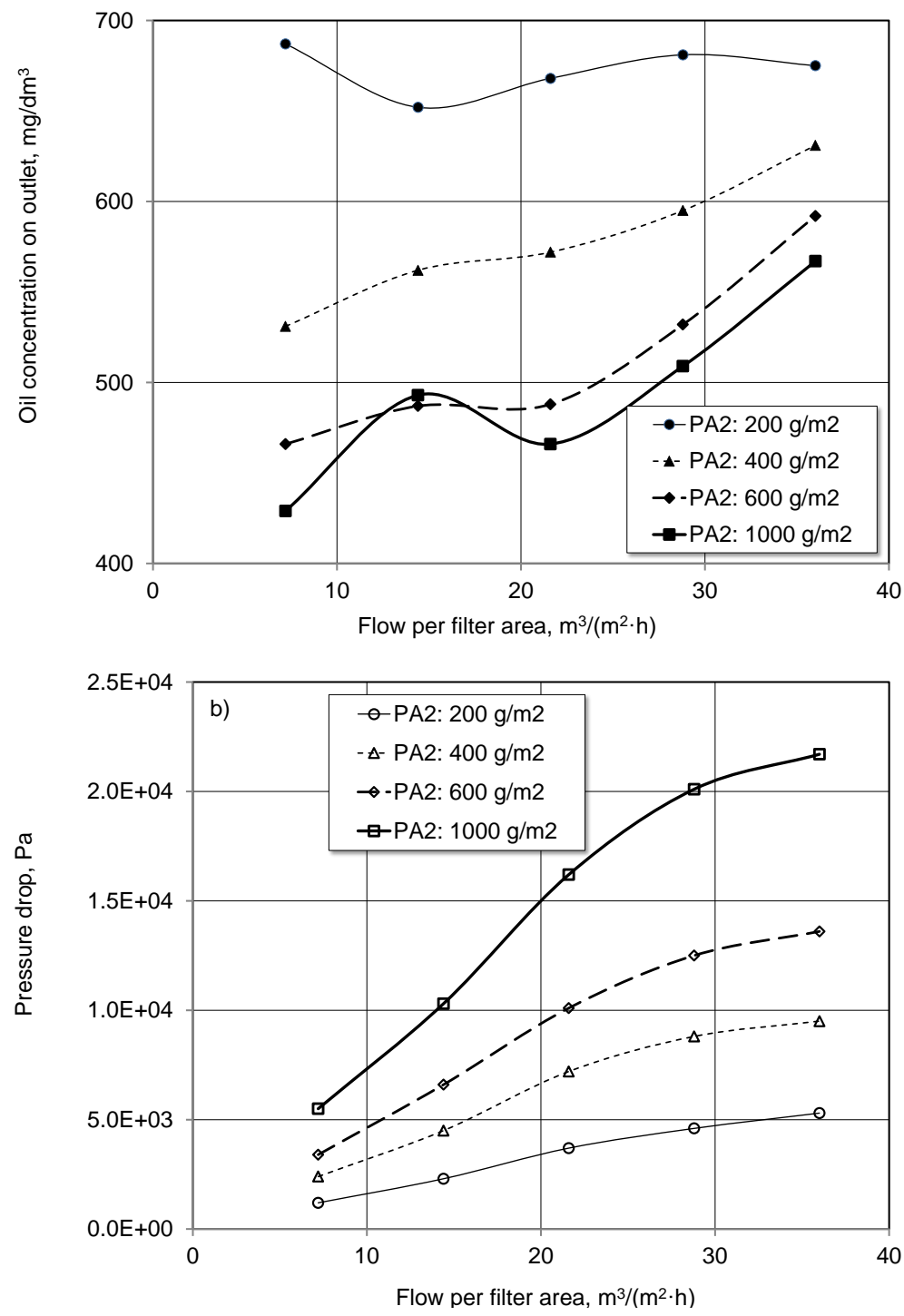

Fig. 10. Outlet oil concentration and pressure drop for coalescence layer made of PA2

This is the main reason why the PA1 with a lower specific surface area performed much worse comparing to PBT1 and PP1 as well (Figs. 5, 7 and 9). The separation experiments confirm that the most hydrophilic polyamide structures are not the best choice for removal of oil from water. Although the pressure drop was the lowest for this polymer, the effluent oil concentration was highest. In addition, a potential risk of swelling, which is typical and commonly known of Nylon ${ }^{\circledR}$ in water, can probably lead to a significant increase of the $\Delta P$ in a long time operation. 
In all the cases, the dependence of pressure drop on the flow rate is not linear as it should be in laminar flow. This can be easily explained by various saturations of the media with oil. The saturation level depends on local hydrodynamics. The higher the local velocity, the less saturated structure is. In other words, the steady-state balance of adhesive force between liquid and fibres, and drag force assisted by gravity pushing the droplets off the fibres is reached at different holdup of accumulated oil. However, at high flow rates and strong adhesion (perfect wetting of fibre surface with oil), a different mechanism of droplet detachment can control the process [22].

Saturation of the porous structure with dispersed phase liquid can be indirectly estimated by measuring the pressure drop for single and two-phase flow. For this purpose a Carman-Kozeny equation can be used:

$$
\frac{\Delta p_{i}}{L}=\frac{36 u \mu_{c} K\left(1-\varepsilon_{i}\right)^{2}}{d_{F}^{2} \varepsilon_{i}^{3}}, \quad i=1,2
$$

where $u$ is the superficial velocity in $\mathrm{m} / \mathrm{s}, L$ depth of a porous bed in $\mathrm{m}, \mu_{c}$ dynamic viscosity of the continuous phase (i.e. water for $\mathrm{O} / \mathrm{W}$ dispersion) in $\mathrm{Pa} \cdot \mathrm{s}, K$ is the Carman-Kozeny constant, and $\varepsilon_{i}$ is the porosity of media for single- $(i=1)$ and two-phase $(i=2)$ flow.

The saturation, i.e. the fraction of voids occupied by dispersed phase, is then obtained from:

$$
S=1-\frac{\varepsilon_{2}}{\varepsilon_{1}}
$$

The saturation is distributed within the porous structure along flow direction [24]. This situation refers, however, to a fibrous bed with a relatively high depth, which is not the case of the present study. Nevertheless, to minimize this effect, the saturation was estimated for $200 \mathrm{~g} / \mathrm{m}^{2}$ media (Fig. 11).

Higher values of the saturation of the coalescence media cause increase of the local shear. This can lead to media plugging and local jetting, but the latter effect has no detrimental impact on separation as long as it refers to the primary coalescence layer. Increased saturation (as observed in the case of PP1) contributes to a shear induced coalescence between free droplets in flow as well as collisions and merging with droplets attached to fibres. However, extensive droplets holdup leads to massive increase of the pressure drop due to filter plugging, which in limiting cases of perfect wetting is usually not balanced by efficiency increase when a quality factor $Q F$ is considered (Table 4). 


$$
Q F=\frac{\ln \left(\frac{c_{\text {in }}}{c_{\text {out }}}\right)}{\Delta P}
$$

Hence, its value does not adequately represent the filter performance, when a low concentration of oil in effluent is the primary objective.

Table 4

Quality factors $Q / F[1 / \mathrm{Pa}]$ for coalescence filters made of various polymers

\begin{tabular}{|c|c|c|c|c|c|c|}
\hline \multirow{3}{*}{$\begin{array}{l}\text { Grammage } \\
\text { of the layer }\end{array}$} & \multicolumn{6}{|c|}{ First layer $[\mathrm{mm} / \mathrm{s}]$} \\
\hline & \multicolumn{2}{|c|}{ PP1 } & \multicolumn{2}{|c|}{ PBT1 } & \multicolumn{2}{|c|}{ PA1 } \\
\hline & 2 & 6 & 2 & 6 & 2 & 6 \\
\hline $200 \mathrm{~g} / \mathrm{m}^{2}$ & 0.405 & 0.165 & 0.627 & 0.250 & 1.421 & 0.570 \\
\hline $400 \mathrm{~g} / \mathrm{m}^{2}$ & 0.226 & 0.093 & 0.343 & 0.144 & 0.762 & 0.324 \\
\hline $600 \mathrm{~g} / \mathrm{m}^{2}$ & 0.152 & 0.062 & 0.251 & 0.108 & 0.535 & 0.235 \\
\hline $1000 \mathrm{~g} / \mathrm{m}^{2}$ & 0.095 & 0.038 & 0.161 & 0.073 & 0.308 & 0.142 \\
\hline
\end{tabular}

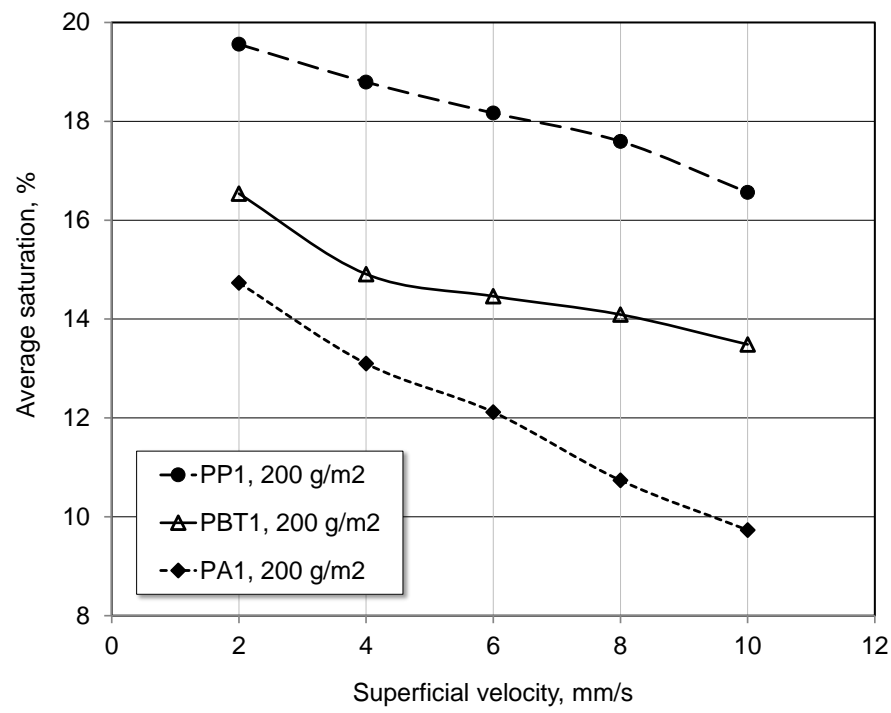

Fig. 11. Saturation of the coalescence layers made of various polymers

The relation between the quality factor and wettability of coalescence media is pronounced: the more oleophilic the fibrous structure, the lower value of QF is. Hence, the $\mathrm{QF}$ definition as commonly used for filters does not give a whole picture about the coalescence performance. 


\subsubsection{EFFECT OF DRAIN LAYER DESIGN}

Four different layers responsible for drainage, i.e. located on the outlet, were tested in a fixed configuration of the primary and secondary coalescence structures. The droplets downstream the coalescence media were photographed and then sized by means of image analysis. The results are presented in Table 5.

Table 5

Effect of properties of the drain layer and flow rate on the size of detached droplets

\begin{tabular}{|c|c|c|c|c|c|}
\hline \multicolumn{4}{|c|}{ Layout and properties of the sandwich structure } & \multicolumn{2}{|c|}{$\begin{array}{l}\text { Size of detached droplets } \\
{[\mathrm{mm}]}\end{array}$} \\
\hline \multicolumn{3}{|c|}{ Grammage (thickness) of the layer } & \multirow{2}{*}{$\begin{array}{c}\text { Mean } \\
\text { fibre } \\
\text { diameter } \\
{[\mu \mathrm{m}]}\end{array}$} & \multirow[b]{2}{*}{ Experimental $^{\mathrm{a}}$} & \multirow[b]{2}{*}{$\begin{array}{c}\text { Model } \\
\text { prediction }^{\mathrm{b}}\end{array}$} \\
\hline Inlet & Intermediate & Drain & & & \\
\hline \multirow{4}{*}{$\begin{array}{c}\text { PP1 } \\
600 \mathrm{~g} / \mathrm{m}^{2} \\
\text { (ca. } 3.5 \mathrm{~mm} \text { ) }\end{array}$} & \multirow{4}{*}{$\begin{array}{c}\text { PP3 } \\
300 \mathrm{~g} / \mathrm{m}^{2} \\
(\text { ca. } 2 \mathrm{~mm})\end{array}$} & $\begin{array}{c}\text { PP4 } \\
400 \mathrm{~g} / \mathrm{m}^{2}(\text { ca. } 5 \mathrm{~mm}) \\
\end{array}$ & 59.5 & $\begin{array}{l}3.7 \pm 2.1^{\mathrm{a}} \\
2.0 \pm 1.6^{\mathrm{b}}\end{array}$ & $\begin{array}{c}1.32^{\mathrm{a}} \\
0.742^{\mathrm{b}}\end{array}$ \\
\hline & & $\begin{array}{c}\text { PP3 } \\
700 \mathrm{~g} / \mathrm{m}^{2}(\text { ca. } 5 \mathrm{~mm}) \\
\end{array}$ & 16.7 & $\begin{array}{l}1.4 \pm 1.3^{\mathrm{a}} \\
1.1 \pm 0.8^{\mathrm{b}}\end{array}$ & $\begin{array}{l}0.834^{\mathrm{a}} \\
0.489^{\mathrm{b}}\end{array}$ \\
\hline & & $\begin{array}{c}\text { PP2 } \\
700 \mathrm{~g} / \mathrm{m}^{2}(\mathrm{ca} .5 \mathrm{~mm}) \\
\end{array}$ & 11.6 & $\begin{array}{l}1.3 \pm 0.8^{\mathrm{a}} \\
1.1 \pm 0.9^{\mathrm{a}} \\
\end{array}$ & $\begin{array}{l}0.811^{\mathrm{a}} \\
0.465^{\mathrm{b}}\end{array}$ \\
\hline & & $\begin{array}{c}\text { PA2 } \\
900 \mathrm{~g} / \mathrm{m}^{2}(\mathrm{ca} .5 \mathrm{~mm}) \\
\end{array}$ & 11.3 & $\begin{array}{l}1.6 \pm 0.4^{\mathrm{a}} \\
1.3 \pm 0.6^{\mathrm{b}}\end{array}$ & $\begin{array}{l}0.912^{\mathrm{a}} \\
0.522^{\mathrm{b}}\end{array}$ \\
\hline
\end{tabular}

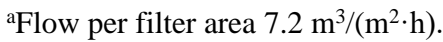

${ }^{b}$ Flow per filter area $36 \mathrm{~m}^{3} /\left(\mathrm{m}^{2} \cdot \mathrm{h}\right)$.

Significant differences were observed for polypropylene layers PP2, PP3 and PP4. The size of detached droplets is strongly affected by size of fibres and flow rate - thick fibre media and low superficial velocities cause an increase of average droplets size downstream the coalescence structure. The PA2 structure performed very well as the drain layer. Comparing to PP2 (characterized by similar average fibre diameter) larger droplets on the outlet were observed. In addition, the deviation around the mean value was very low, which means that very uniform droplets were detached from the coalescence structure. The droplet size measured in experiments was compared with prediction of the numerical model (last column in Table 5), which includes two independent criteria of detachment taking into account local hydrodynamics and surface properties of fibres. The differences of predicted size of detached droplets arise from simplification assumed in the model with regards to the fibre-droplet geometry [22]. In the presented approach, the virtual droplet is attached to a single fibre, while in real situation it can be in contact with a number of fibres, e.g. at fibre intersections. In such cases, the force of adhesion is much stronger due to the larger wetting line, which leads to larger size of detached droplets as observed experimentally. Nevertheless, the experiments confirm 
that the tendencies predicted by the model are correct. The model explains importance of media wettability with regards to dispersed phase liquid (i.e. oil in the case of oil-in-water dispersions). When detachment is governed by force balance, i.e. adhesion and viscous drag, the operation of coalescence media is stable. This mechanism controls disengagement of droplets which are large and uniform in size. Such situation is likely to occur at moderate or weak wettability. On the other hand, for perfect wettability the mechanism of detachment can be different. Due to strong adhesion, the structure saturation is very high and very tight passages for flow are available within the porous structure. In such a case, the viscous shear is very high and there is a high probability of jetting and redispersion. Then the droplets can potentially be detached due to an uncontrolled and random breakup process. As a result, a high variation of droplet size around the average value can be observed on the outlet as in the case of polypropylene media (Table 5).

\section{CONCLUSIONS}

In this work, the performance of multilayer coalescence media was studied. The results of separation efficiency show that there is a critical depth (or grammage) of fibrous structure, above which no further decrease of outlet oil concentration was observed. For fibrous media made of relatively thick fibres (PP2, PBT2, PA2), the maximum coalescence efficiency is approached at much lower grammage.

To determine the effect of the fibre wettability in the coalescence layer (located on the inlet), the assembled structures were very similar in design but made of different materials. For studied polymers, the highest separation efficiency was achieved for strongly oleophilic polypropylene media PP1 with very fine fibres. However, the good wetting caused strong adhesion and led to plugging of the filter with the dispersed phase liquid. Such extensive saturation resulted in a massive increase of the pressure drop.

The effect of flow rate on separation efficiency was not very significant. In the case of thin fibre media PP1 and PBT1, a small decrease of the oil concentration on the outlet can be explained by a low dynamic stability of the structure and its compaction at high $\Delta \mathrm{P}$.

Moreover, the effect of the drain layer design was verified. The experiments confirmed the tendencies obtained from numerical model, which was developed for single cell representing the fibrous media. The size of detached droplets is strongly dependent on fibre diameter - increase of the fibre diameter and decrease of the flow rate led to detachment of larger droplets. An important conclusion can be drawn from the study related to effect of wettability of the drain layer media on the size of droplets released from the drain layer. Even for PP4 structure consisted of the thickest tested fibres (average diameter around $60 \mu \mathrm{m}$ ), the spread of detached droplet size was significant. One should bear in mind that droplets breakup is a random process and unlike in the model 
(where symmetrical breakup is assumed) fine secondary droplets can be present on the outlet. Therefore, the design of the drainage media should enable fulfilling detachment criterion due to force balance rather than breaking the droplets when the critical Weber number is exceeded. First criterion governs the drainage process when drag force resulting from the bulk flow and assisted by gravity exceeds force of adhesion of accumulated liquid to fibres (as it probably could be in the case of polyamide media, which are less oleophilic than polypropylene in surrounding water). In the case of drain layer made of PA2, the droplets observed downstream the filter were much more uniform in size than released from polypropylene fibres.

When compared to the state-of-art separation of oil from water using modified hydrophilic-oleophobic membranes, the oil removal efficiency by coalescence filtration is lower with regards to small droplets of the size approaching $1 \mu \mathrm{m}$ and below. However, above $2-3 \mu \mathrm{m}$ deep bed filtration with properly designed coalescence structure guarantees a comparable residual oil concentration in the effluent. Hence, the coalescers can be successfully applied and be competitive to membrane processes for dispersions characterized by micron-sized droplets, i.e. created by flow through low or moderate energy devices. The main limitation for application the membrane separation is a very high cost. In addition, due to the fact that coalescers operate at higher face velocities, much less coalescence surface is required comparing to membrane separation, and this also reflects the cost. Therefore, the operation cost (lower pressure drop) as well as capital investment related to equipment size and cost of internals are both in favour to coalescence filtration.

\section{ACKNOWLEDGEMENTS}

This work was supported by Polish National Science Centre, grant No. N N209 029340. The author wishes to thank Mr. Witalis Rumiński from the Amazon Filters Sp. z o.o. for preparing the fibrous structures.

\section{REFERENCES}

[1] DAVIES G.A., JefFreys G.V., Coalescence of droplets in packings: Factors affecting the separation of droplet dispersions, Filtr. Sep., 1969, 6, 4, 349.

[2] SHERONy D.F., KinTner R.C., WASAN D.T., Coalescence of secondary emulsions in fibrous beds, Surf. Coll. Sci., 1978, 10, 99.

[3] ABDEL-GHANi M.S., Coalescence and flow of secondary dispersions in fibre beds, $\mathrm{PhD}$ thesis, University of Manchester, UK, 1983.

[4] Clayfield A.J., DiXON A.G., Foulds A.W., MilleR R.J.L., The coalescence of secondary dispersions. I. The effect of wettability and surface energy, J. Coll. Int. Sci., 1985, 104, 2, 500.

[5] Othman F.M., Fahim M.A., JefFreys G.V., MumFord C.J., Prediction of predominant mechanisms in the separation of secondary dispersions in a fibrous bed, J. Disp. Sci. Technol., 1988, 9, $2,91$.

[6] Magiera R., Blass E., Separation of liquid-liquid dispersions by flow through fibre beds, Filtr. Sep., 1997, 34, 4, 369. 
[7] SECEROV-SOKOlovic R.M., SOKOlovic S.M., Effect of the nature of different polymeric fibers on steady-state bed coalescence of an oil-in-water emulsion, Ind. Eng. Chem. Res., 2004, 43, 6490.

[8] SHIN C., ChASE G.G., Separation of water-in-oil emulsions using glass fiber media augmented with polymer nanofibers, J. Disp. Sci. Technol., 2006, 27, 4, 517.

[9] Secerov-Sokolovic R.M., Vulic T.J., Sokolovic S.M., Effect of bed length on steady-state coalescence of oil-in-water emulsion, Sep. Purif. Technol., 2007, 56, 79.

[10] Bansal S., Von ARnim V., Stegmaier T., Planck H., Effect of fibrous filter properties on the oil-inwater-emulsion separation and filtration performance, J. Hazard. Mat., 2011, $190,45$.

[11] Agarval S., Von Arnim V., Stegmaier T., Planck H., Agarwal A., Role of surface wettability and roughness in emulsion separation, Sep. Purif. Technol., 2013, 107, 19.

[12] KUNDU P., MishRA I.M., Removal of emulsified oil from oily wastewater (oil-in-water emulsion) using packed bed of polymeric resin beads, Sep. Purif. Technol., 2013, 118, 519.

[13] Maiti S., Mishra I.M., BhatTACHARYA S.D., Joshi J.K., Removal of oil from oil-in-water emulsion using a packed bed of commercial resin, Coll. Surf. A: Phys. Eng. Asp., 2011, 389, 291.

[14] ZHU Y., WANG D., JIANG L., JIN J., Recent progress in developing advanced membranes for emulsified oil/water separation, NPG Asia Mat., 2014, 6, 101.

[15] Solomon B.R., Hyder M.N., VARANASI K.K., Separating oil-water nanoemulsions using flux-enhanced hierarchical membranes, Sci. Rep., 2014, 4, 5504, 1.

[16] PAtel S.U., ChaSe G.G., Separation of water droplets from water-in-diesel dispersion using superhydrophobic polypropylene fibrous membranes, Sep. Purif. Technol., 2014, 126, 62.

[17] GoGóŁ W., Heat exchange. Tables and Graphs, Wyd. Politechniki Warszawskiej, Warszawa 1984.

[18] GöBel J.G., JopPIEN G.R., Dynamic interfacial tensions of aqueous Triton X-100 solutions in contact with air, cyclohexane, n-heptane, and n-hexadecane, J. Colloid Interf. Sci., 1997, 191, 30.

[19] Polish Standard Test Method PN-82/C.04565.01: Water and Wastewater-Determination of the nonpolar aliphatic hydrocarbons using IR spectroscopy.

[20] Siebold A., NARdin M., SchUltz J., WAlliser A., OpPliger M., Effect of dynamic contact angle on capillary rise phenomena, Colloids Surf., A: Physicochem. Eng. Asp., 2000, 161, 81.

[21] YANG B.W., ChANG Q., Wettability studies of filter media using capillary rise test, Sep. Purif. Technol., 2008, 60, 335.

[22] http://www.accudynetest.com/polytable_01.html

[23] KRASINSKI A., A numerical model of droplets coalescence and drainage in fibrous structures, Chem. Eng. Trans., 2013, 32, 1495.

[24] Gutkowski B., Mydlarczyk S., Kowalska M., HupKa J., Saturation profiles in coalescence bed, Stud. Env. Sci., 1984, 23, 285. 